\title{
A New Kumaraswamy Class of Generalized Distributions with Applications to Exponential Model
}

\author{
Mohamed Ali AHMED1,*
}

${ }^{1}$ Department of Statistics, Mathematics and Insurance, Al Madina Higher Institute of Management and Technology, Giza, Egypt, mrmohamedali2005@yahoo.com Orcid: 0000-0002-8320-6631 ${ }^{1}$

*Correspondence Author, e-mail: mrmohamedali2005@yahoo.com

\begin{abstract}
In this paper, a new class of generalized distributions, so-called the alpha power Kumaraswamy $(A K)$ class, is derived, three important classes of distributions are nested by the $A K$ class. Some mathematical properties are studied and a parameters estimation method using maximum likelihood (MLE) is obtained. A simulation study using bootstrapping approach is applied to study the alpha power Kumaraswamy-exponential (AKE) distribution estimators' behavior. A real data set is used to investigate the AKE distribution flexibility.

Keywords: the alpha power Kumarasamy distribution; moments; order statistics; maximum likelihood estimation; bootstrapping approach.
\end{abstract}

\section{Introduction}

Adding parameters using alpha power transformation $(A P T)$ is a flexible method depending on the alpha power function, [1], having the following cumulative distribution function $(C D F)$ and probability density function $(P D F)$ for a continuous random variable $X$, respectively

$F_{A P T}(x)=\left\{\begin{array}{l}\frac{\alpha^{F(x)}-1}{\alpha-1} ; \alpha>0, \alpha \neq 1, \\ F(x) ; \alpha=1,\end{array}\right.$

and

$f_{A P T}(x)=\left\{\begin{array}{l}\frac{\log \alpha}{\alpha-1} f(x) \alpha^{F(x)} ; \alpha>0, \alpha \neq 1, \\ f(x) ; \alpha=1 .\end{array}\right.$

Ahmed [2] presented for the first time the $A K$ distribution having the following $C D F$ and $P D F$, respectively,

$$
F_{\alpha K}(x)=\frac{\alpha^{1-\left(1-x^{\beta}\right)^{\theta}}-1}{\alpha-1}, 0<x<1 ; \alpha, \beta, \theta>0 ; \alpha \neq 1 \text {, }
$$

and 


$$
f_{\alpha K}(x)=\frac{\beta \theta \log \alpha}{\alpha-1} \alpha^{1-\left(1-x^{\beta}\right)^{\theta}} x^{\beta-1}\left(1-x^{\beta}\right)^{\theta-1} .
$$

The main object of this manuscript is to derive an extended class of generalized distributions naming the $A K$ class of generalized distributions depending on the $A K$ distribution, Ahmed [2], as a simple generator, also it aims to study some properties of the class and gives some applications to $A K E$ distribution.

The rest of this paper is organized as follows: In section 2, the generalized class is presented. In section 3 , some properties are derived. In section 4, the Hazard function is given. In section 5, order statistics are obtained. In Section 6, the MLE method is used. In section 7, a simulation study using bootstrapping is performed for the $A K E$ distribution. Finally, in Section 8, an application is investigated, practically, for the $A K E$ distribution.

\section{The New Class of $A K$ Distributions}

Many classes of generalized distributions are derived based on generating method, Wahed [3], the Kumaraswamy $(K W)$ class [4] and [5], the Kummer beta class [6], the McDonald class [7] and [8], the Kumaraswamy- Kumaraswamy (KW-KW) class [9] and [10]. Replacing $x$ in (1) with the generalized parent $G(x ; \Lambda)$ gives

$$
F(x)=\frac{\alpha^{1-\left(1-G^{\beta}(x ; \Lambda)\right)^{\theta}}-1}{\alpha-1} ; \alpha, \beta, \theta>0 ; \alpha \neq 1,
$$

differentiating (3) with respect to $x$ yields

$$
f(x)=\frac{\beta \theta}{\alpha-1}(\log \alpha) \alpha^{1-\left(1-G^{\beta}(x ; \Lambda)\right)^{\theta}} g(x ; \Lambda) G^{\beta-1}(x ; \Lambda)\left(1-G^{\beta}(x ; \Lambda)\right)^{\theta-1},
$$

where $G(x ; \Lambda)$ and $g(x ; \Lambda)$ are the $C D F$ and $p d f$ of the parent distribution, $\Lambda$ is the parameter vector of the parent distribution. When $\alpha=1$, the $A K$ class gives Kumaraswamy (Kw) class [4] and [5], setting $\theta=1$ gives the alpha power $(A P)$ class and setting $\alpha=1, \theta=1$ gives the power function $(P)$ class [11]. Many distributions can be derived via the class of $A K$ as the alpha power Kumaraswamy exponential ( $A K E$ ) distribution, the $C D F$ and $P D F$ of the $A K E$ distribution, respectively, can be given by

$$
F(x)=\frac{\alpha^{1-\left[1-\left(1-e^{-\lambda x}\right)^{\beta}\right]^{\theta}}-1}{\alpha-1} ; \alpha, \beta, \theta, \lambda>0 ; \alpha \neq 1,
$$

and

$$
f(x)=\frac{\beta \theta \lambda}{\alpha-1}(\log \alpha) \alpha^{1-\left(1-\left(1-e^{-\lambda x}\right)^{\beta}\right)^{\theta}} e^{-\lambda x}\left(1-e^{-\lambda x}\right)^{\beta-1}\left(1-\left(1-e^{-\lambda x}\right)^{\beta}\right)^{\theta-1},
$$

some density function shapes for the $A K E$ distribution are given in figure 1.

\subsection{The CDF Expansion}

Applying the exponential expansion for (3) leads to

$$
F(x)=\frac{1}{1-\alpha}\left\{1-\sum_{i=0}^{\infty} \frac{\left[1-\left(1-G^{\beta}(x ; \Lambda)\right)^{\theta}\right]^{i}(\log \alpha)^{i}}{i !}\right\}, \alpha, \beta, \theta>0 ; \alpha \neq 1,
$$


then, using binomial expansion gives

$$
F(x)=\frac{1}{1-\alpha}\left\{1-\sum_{i=0}^{\infty} \frac{(\log \alpha)^{i}}{i !} \sum_{j=0}^{i}(-1)^{j}\left(\begin{array}{l}
i \\
j
\end{array}\right)\left(1-G^{\beta}(x ; \Lambda)\right)^{\theta j}\right\},
$$

replacing $\sum_{i=0}^{\infty} \sum_{j=0}^{i}$ with $\sum_{j=0}^{\infty} \sum_{i=j}^{\infty}$ yields

$$
F(x)=\frac{1}{1-\alpha}\left\{1-\sum_{j=0}^{\infty} \sum_{i=j}^{\infty} \frac{(\log \alpha)^{i}}{i !}(-1)^{j}\left(\begin{array}{l}
i \\
j
\end{array}\right)\left(1-G^{\beta}(x ; \Lambda)\right)^{\theta j}\right\},
$$

using binomial expansion, again, leads to

$$
F(x)=\frac{1}{1-\alpha}\left\{1-\sum_{j=0}^{\infty} \sum_{i=j}^{\infty} \sum_{k=0}^{\infty}(-1)^{j+k} \frac{(\log \alpha)^{i}}{i !}\left(\begin{array}{c}
i \\
j
\end{array}\right)\left(\begin{array}{c}
\theta j \\
k
\end{array}\right) G^{\beta k}(x ; \Lambda)\right\},
$$

where $\beta$ is an integer, when $\beta$ is real non integer yields

$$
F(x)=\frac{1}{1-\alpha}\left\{1-\sum_{j=0}^{\infty} \sum_{i=j}^{\infty} \sum_{k=0}^{\infty}(-1)^{j+k} \frac{(\log \alpha)^{i}}{i !}\left(\begin{array}{c}
i \\
j
\end{array}\right)\left(\begin{array}{c}
\theta j \\
k
\end{array}\right)[1-(1-G(x ; \Lambda))]^{\beta k}\right\},
$$

then, using binomial expansion two times gives

$$
F(x)=\frac{1}{1-\alpha}\left\{1-\sum_{j=0}^{\infty} \sum_{i=j}^{\infty} \sum_{k=0}^{\infty} \sum_{p=0}^{\infty} \sum_{q=0}^{p}(-1)^{j+k+p+q} \frac{(\log \alpha)^{i}}{i !}\left(\begin{array}{c}
i \\
j
\end{array}\right)\left(\begin{array}{c}
\theta j \\
k
\end{array}\right)\left(\begin{array}{c}
\beta k \\
p
\end{array}\right)\left(\begin{array}{l}
p \\
q
\end{array}\right) G^{q}(x ; \Lambda)\right\},
$$

replacing $\sum_{p=0}^{\infty} \sum_{q=0}^{p}$ with $\sum_{q=0}^{\infty} \sum_{p=q}^{\infty}$ leads to

$$
F(x)=\frac{1}{1-\alpha}\left\{1-\sum_{q=0}^{\infty} w_{q} G^{q}(x ; \Lambda)\right\},
$$

where

$$
w_{q}=\sum_{j=0}^{\infty} \sum_{i=j}^{\infty} \sum_{k=0}^{\infty} \sum_{p=q}^{\infty}(-1)^{j+k+p+q} \frac{(\log \alpha)^{i}}{i !}\left(\begin{array}{c}
i \\
j
\end{array}\right)\left(\begin{array}{c}
\theta j \\
k
\end{array}\right)\left(\begin{array}{c}
\beta k \\
p
\end{array}\right)\left(\begin{array}{l}
p \\
q
\end{array}\right) .
$$

\subsection{The PDF Expansion}

Differentiating (5) with respect to $x$ gives

$$
f(x)=\frac{1}{1-\alpha}\left\{\sum_{q=1}^{\infty} w_{q} q G^{q-1}(x ; \Lambda) g(x ; \Lambda)\right\},
$$

shifting $q$ leads to

$$
f(x)=\frac{1}{1-\alpha}\left\{\sum_{q=0}^{\infty} w_{q+1}(q+1) G^{q}(x ; \Lambda) g(x ; \Lambda)\right\},
$$

then, 


$$
f(x)=\sum_{q=0}^{\infty} w_{q}^{*} G^{q}(x ; \Lambda) g(x ; \Lambda),
$$

where

$$
w_{q}^{*}=\frac{1}{1-\alpha} w_{q+1}(q+1)
$$

\section{The Condition for the PDF Expansion}

since,

$$
\sum_{q=0}^{\infty} w_{q}^{*} \int_{-\infty}^{\infty} G^{q}(x ; \Lambda) g(x ; \Lambda) d x=1,
$$

then,

$$
\sum_{q=0}^{\infty} w_{q}^{*}\left[\frac{G^{q+1}(x ; \Lambda)}{(q+1)}\right]_{-\infty}^{\infty}=1
$$

hence,

$$
\sum_{q=0}^{\infty} \frac{w_{q}^{*}}{q+1}=1
$$
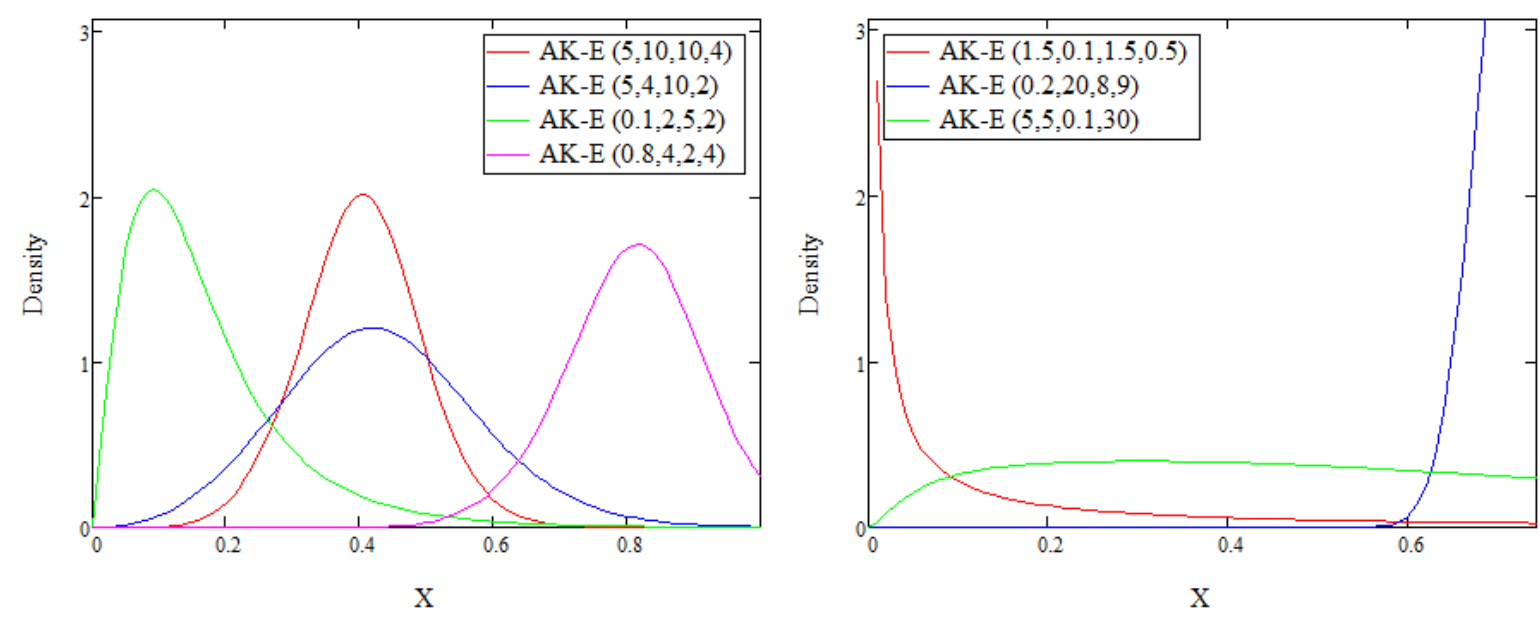

Figure 1: The $A K E$ density functions

\section{The $A K$ Class of Distributions Properties}

In this section some properties of the $A K$ class of distributions will be given as follows:

\subsection{The r-th Moment}

Basically, a continuous random variable $X$ has the following $r$-th moment [12]

$$
E\left(X^{r}\right)=\int_{x} x^{r} f(x) d x
$$

substituting (6) into last equation yields 


$$
E\left(X^{r}\right)=\sum_{q=0}^{\infty} w_{q}^{*} \int_{-\infty}^{\infty} x^{r} g(x ; \Lambda) G^{q}(x ; \Lambda) d x
$$

then,

$$
E\left(X^{r}\right)=\sum_{q=0}^{\infty} w_{q}^{*} \tau_{r, q, 0}
$$

where $\tau$ is the probability weighted moment (PWM), Greenwood et. al. [13].

Obviously, setting $r=0$ and using (9) leads to

$$
E\left(X^{0}\right)=\sum_{q=o}^{\infty} w_{q}^{*} \int_{-\infty}^{\infty} g(x ; \Lambda) G^{q}(x ; \Lambda) d x
$$

then,

$$
E\left(x^{0}\right)=\sum_{q=o}^{\infty} w_{q}^{*}\left[\frac{G^{q+1}(x ; \Lambda)}{q+1}\right]_{-\infty}^{\infty},
$$

substituting (8) into last equation gives

$$
E\left(X^{0}\right)=1
$$

\section{Using the Parent Quantile Function}

Setting $G(x ; \Lambda)=u, x=Q(u)$ and substituting into (9) gives

$$
E\left(X^{r}\right)=\sum_{q=o}^{\infty} w_{q}^{*} \int_{0}^{1} Q^{r}(u) u^{q} d u
$$

then,

$$
E\left(X^{r}\right)=\sum_{q=o}^{\infty} w_{q}^{*} \tau_{r, q, o}
$$

\subsection{The PWM}

Basically, the $P W M$ of a continuous random variable $X$, Greenwood et. al. [13], is given by

$$
\tau_{r, \mathrm{~s}, 0}=\int_{x} x^{r} f(x) F^{s}(x) d x,
$$

substituting (5) and (6) into last equation leads to

$$
\tau_{r, s, o}=\left(\frac{1}{1-\alpha}\right)^{s} \int_{-\infty}^{\infty} x^{r} g(x ; \Lambda)\left[\sum_{q=o}^{\infty} w_{q}^{*} G^{q}(x ; \Lambda)\right]\left[1-\sum_{q=0}^{\infty} w_{q} G^{q}(x ; \Lambda)\right]^{s} d x,
$$

using binomial expansion yields 


$$
\tau_{r, s, o}=\left(\frac{1}{1-\alpha}\right)^{s} \sum_{k=0}^{s}(-1)^{k}\left(\begin{array}{l}
s \\
k
\end{array}\right) \int_{-\infty}^{\infty} x^{r} g(x ; \Lambda)\left[\sum_{q=o}^{\infty} w_{q}^{*} G^{q}(x ; \Lambda)\right]\left[\sum_{q=0}^{\infty} w_{q} G^{q}(x ; \Lambda)\right]^{k} d x,
$$

since, $\left[\sum_{q=0}^{\infty} w_{q} G^{q}(x ; \Lambda)\right]^{k}=\left[\sum_{q=0}^{\infty} c_{q} G^{q}(x ; \Lambda)\right]$, Gradshteyn and Ryzhik [14], then,

$$
\tau_{r, s, o}=\left(\frac{1}{1-\alpha}\right)^{s} \sum_{k=0}^{s}(-1)^{k}\left(\begin{array}{l}
s \\
k
\end{array}\right) \int_{-\infty}^{\infty} x^{r} g(x ; \Lambda)\left[\sum_{q=o}^{\infty} w_{q}^{*} G^{q}(x ; \Lambda)\right]\left[\sum_{q=0}^{\infty} c_{q} G^{q}(x ; \Lambda)\right] d x,
$$

where

$$
c_{0}=w_{0}^{k}, c_{m}=\frac{1}{m w_{0}} \sum_{q=1}^{m}(q k-m+q) w_{q} c_{m-q} ; m \geq 1,
$$

since, $\left[\sum_{q=o}^{\infty} w_{q}^{*} G^{q}(x ; \Lambda)\right]\left[\sum_{q=0}^{\infty} c_{q} G^{q}(x ; \Lambda)\right]=\sum_{q=o}^{\infty} d_{q} G^{q}(x ; \Lambda)$, Gradshteyn and Ryzhik [14],

hence,

$$
\tau_{r, s, o}=\left(\frac{1}{1-\alpha}\right)^{s} \sum_{k=0}^{s}(-1)^{k}\left(\begin{array}{l}
s \\
k
\end{array}\right) \int_{-\infty}^{\infty} x^{r} g(x ; \Lambda)\left[\sum_{q=o}^{\infty} d_{q} G^{q}(x ; \Lambda)\right] d x
$$

where

$$
d_{m}=\sum_{q=0}^{m} w_{q}^{*} c_{m-q}
$$

since,

$$
\tau_{r, s, o}=\sum_{q=o}^{\infty} h_{q} \int_{-\infty}^{\infty} x^{r} g(x ; \Lambda) G^{q}(x ; \Lambda) d x,
$$

where

$$
h_{q}=\left(\frac{1}{1-\alpha}\right)^{s} \sum_{k=0}^{s} \sum_{q=o}^{\infty} d_{q}(-1)^{k}\left(\begin{array}{l}
s \\
k
\end{array}\right) \text {, }
$$

then,

$$
\tau_{r, s, o}=\sum_{q=o}^{\infty} h_{q} \tau_{r, q, o}
$$

\section{Using the Parent Quantile Function}

Setting $G(x ; \Lambda)=u, y=Q(u)$ and substituting into (11) yields

$$
\tau_{r, s, o}=\sum_{q=o}^{\infty} h_{q} \int_{0}^{1} Q^{r}(u) u^{q} d u
$$


then,

$$
\tau_{r, s, o}=\sum_{q=o}^{\infty} h_{q} \tau_{r, q, o}
$$

\subsection{The Moment Generating Function}

A continuous random variable $X$ moment generating function $(M G F)$ can be written as

$$
M_{x}(t)=E\left(e^{t x}\right)=\int_{x} e^{t x} f(x) d x
$$

applying the exponential expansion yields

$$
E\left(e^{t x}\right)=E\left(\sum_{r=0}^{\infty} \frac{t^{r} x^{r}}{r !}\right)
$$

then,

$$
E\left(e^{t x}\right)=\sum_{r=0}^{\infty} \frac{t^{r} E\left(x^{r}\right)}{r !}
$$

substituting (10) into last equation gives

$$
E\left(e^{t x}\right)=\sum_{r=0}^{\infty} \sum_{q=0}^{\infty} \frac{t^{r}}{r !} w_{q}^{*} \tau_{r, q, 0} .
$$

\section{Using the Parent Quantile Function}

Substituting (6) into (12) leads to

$$
E\left(e^{t x}\right)=\int_{-\infty}^{\infty} e^{t x} \sum_{q=0}^{\infty} w_{q}^{*} G^{q}(x ; \Lambda) g(x ; \Lambda) d x,
$$

then,

$$
E\left(e^{t x}\right)=\sum_{q=0}^{\infty} w_{q}^{*} \int_{-\infty}^{\infty} e^{t x} G^{q}(x ; \Lambda) g(x ; \Lambda) d x
$$

setting $G(x ; \Lambda)=u, x=Q(u)$ and substituting into last equation yields

$$
E\left(e^{t x}\right)=\sum_{q=o}^{\infty} w_{q}^{*} \int_{0}^{1} e^{t Q(u)} u^{q} d u
$$

using exponential expansion gives

$$
E\left(e^{t x}\right)=\sum_{q=o}^{\infty} w_{q}^{*} \sum_{r=0}^{\infty} \frac{t^{r}}{r !} \int_{0}^{1} Q^{r}(u) u^{q} d u,
$$

hence, 


$$
E\left(e^{t x}\right)=\sum_{q=o}^{\infty} w_{q}^{*} \sum_{r=0}^{\infty} \frac{t^{r}}{r !} \tau_{r, q, 0} .
$$

\subsection{The Mean Deviation}

Basically, a random variable $X$ having the mean deviation about mean and median, respectively, can be written as

$$
S_{1}(x)=\int_{x}|x-\mu| f(x) d x \quad \text { and } \quad S_{2}(x)=\int_{x}|x-M| f(x) d x,
$$

which is given by, Ali Ahmed [15],

$$
S_{1}(x)=2 \mu F(\mu)-2 t(\mu) \text { and } S_{2}(x)=\mu-2 t(M),
$$

where $T(z)=\int_{-\infty}^{z} x f(x) d x$ is the linear incomplete moment.

Substituting (6) into T(.) yields

$$
T(z)=\int_{-\infty}^{z} x \sum_{q=o}^{\infty} w_{q}^{*} g(x ; \Lambda) G^{q}(x ; \Lambda) d y,
$$

then,

$$
T(z)=\sum_{q=o}^{\infty} w_{q}^{*} \int_{-\infty}^{z} x g(x ; \Lambda) G^{q}(x ; \Lambda) d y .
$$

\section{Using the Parent Quantile Function}

Setting $G(x ; \Lambda)=u, x=Q(u)$ and substituting into last equation gives

$$
T(z)=\sum_{q=o}^{\infty} w_{p}^{*} \int_{0}^{G(z)} Q(u) u^{q} d u .
$$

\section{The Hazard Function of the MLN Class of Distributions}

A random variable $X$ survival function [16] can be written as

$$
S(x)=1-F(x)
$$

substituting (3) into last equation gives

$$
S(x)=\frac{\alpha-\alpha^{1-\left(1-G^{\beta}(x ; \Lambda)\right)^{\theta}}}{\alpha-1} ; \alpha, \beta, \theta>0 ; \alpha \neq 1,
$$

moreover, the Hazard function [16] can be written as

$$
H(x)=\frac{f(x)}{S(x)},
$$


substituting (4) and (13) into last equation yields

$$
H(x)=\frac{\beta \theta(\log \alpha) \alpha^{1-\left(1-G^{\beta}(x ; \Lambda)\right)^{\theta}} g(x ; \Lambda) G^{\beta-1}(x ; \Lambda)\left(1-G^{\beta}(x ; \Lambda)\right)^{\theta-1}}{\alpha-\alpha^{1-\left(1-G^{\beta}(x ; \Lambda)\right)^{\theta}}} .
$$

The AKE Hazard function can be given by

$$
H(x)=\frac{\beta \theta \lambda(\log \alpha) \alpha^{1-\left(1-\left(1-e^{-\lambda x}\right)^{\beta}\right)^{\theta}} e^{-\lambda x}\left(1-e^{-\lambda x}\right)^{\beta-1}\left(1-\left(1-e^{-\lambda x}\right)^{\beta}\right)^{\theta-1}}{\alpha-\alpha^{1-\left(1-\left(1-e^{-\lambda x}\right)^{\beta}\right)^{\theta}}},
$$

some Hazard function shapes for the AKE distribution are given in figure 2.

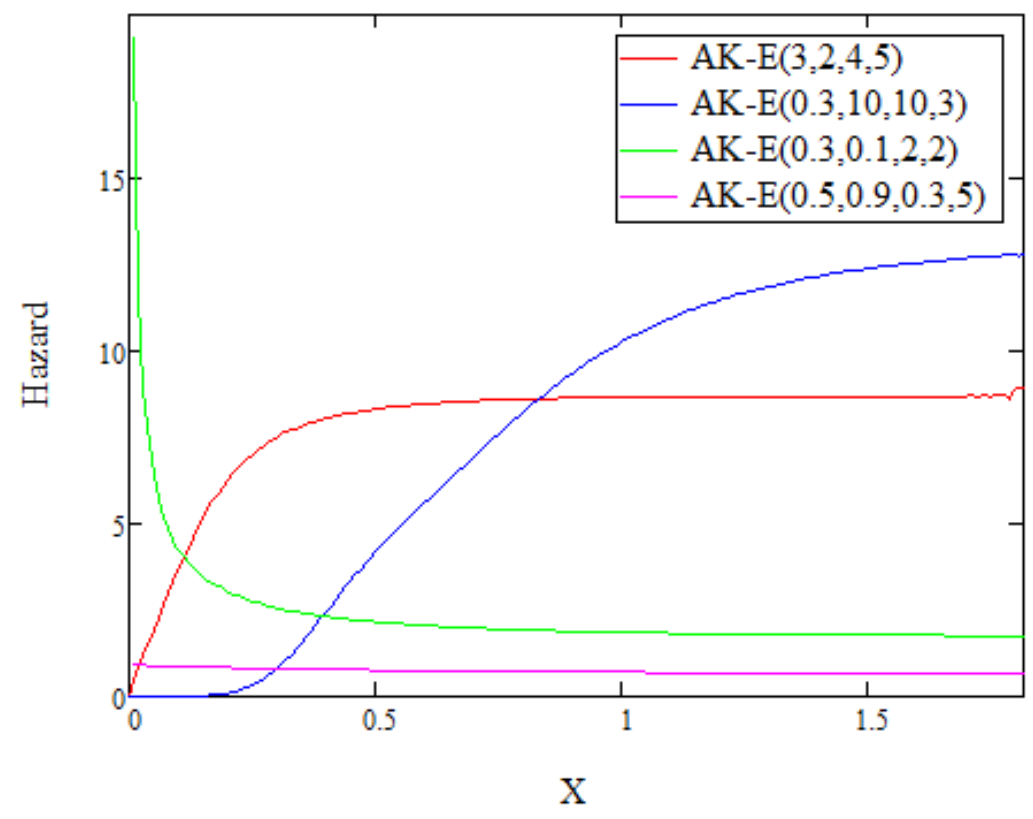

Figure 2: The $A K E$ Hazard functions

In figure 2, Hazard functions curves four types for the $A K E$ distribution are illustrated as follows: $\mathrm{A}$ decreasing then constant Hazard curve, a constant Hazard curve, an increasing then constant Hazard curve and a constant then increasing then constant Hazard curve.

\section{Order Statistics of the $A K$ Class of Distributions}

The $u$-th order statistics density function $f\left(x_{u: v}\right)$ for $u=1,2, \ldots, v$ from iid random variables $X_{1}, X_{2}, \ldots, X_{v}$ following any $A K$ generalized distribution [17] can be written as

$$
f\left(x_{u: v}\right)=\frac{f\left(x_{u}\right)}{B(u, v-u+1)} F^{u-1}\left(x_{u}\right)\left\{1-F\left(x_{u}\right)\right\}^{v-u},
$$

applying binomial expansion for the last equation leads to 


$$
f\left(x_{u v}\right)=\sum_{p=0}^{v-u} \frac{(-1)^{p}\left(\begin{array}{c}
v-u \\
p
\end{array}\right)}{B(u, v-u+1)} f\left(x_{u}\right) F^{u+p-1}\left(x_{u}\right)
$$

substituting (5) and (6) into last equation leads to

$f\left(x_{u v}\right)=\sum_{p=0}^{v-u} \frac{(-1)^{p}\left(\begin{array}{c}v-u \\ p\end{array}\right)}{B(u, v-u+1)}\left[\sum_{q=0}^{\infty} w_{q}^{*} G^{q}\left(x_{u} ; \Lambda\right) g\left(x_{u} ; \Lambda\right)\right] \times\left[\frac{1}{1-\alpha}\left(1-\sum_{q=0}^{\infty} w_{q} G^{q}\left(x_{u} ; \Lambda\right)\right)\right]^{u+p-1}$, then,

$f\left(x_{u v v}\right)=\sum_{p=0}^{v-u} \frac{(-1)^{p}\left(\begin{array}{c}v-u \\ p\end{array}\right)\left(\frac{1}{1-\alpha}\right)^{u+p-1}}{B(u, v-u+1)}\left[\sum_{q=0}^{\infty} w_{q}^{*} G^{q}\left(x_{u} ; \Lambda\right) g\left(x_{u} ; \Lambda\right)\right]\left[1-\sum_{q=0}^{\infty} w_{q} G^{q}\left(x_{u} ; \Lambda\right)\right]^{u+p-1}$,

using binomial expansion gives

$$
\begin{aligned}
f\left(x_{u v}\right) & =\sum_{p=0}^{v-u} \frac{(-1)^{p}\left(\begin{array}{c}
v-u \\
p
\end{array}\right)\left(\frac{1}{1-\alpha}\right)^{u+p-1}}{B(u, v-u+1)}\left[\sum_{q=0}^{\infty} w_{q}^{*} G^{q}\left(x_{u} ; \Lambda\right) g\left(x_{u} ; \Lambda\right)\right] \\
& \times \sum_{s=0}^{u+p-1}(-1)^{s}\left(\begin{array}{c}
u+p-1 \\
s
\end{array}\right)\left(\sum_{q=0}^{\infty} w_{q} G^{q}\left(x_{u} ; \Lambda\right)\right)^{s},
\end{aligned}
$$

since, $\left(\sum_{q=0}^{\infty} w_{q} G^{q}\left(x_{u} ; \Lambda\right)\right)^{s}=\left[\sum_{q=0}^{\infty} a_{q} G^{q}\left(x_{u} ; \Lambda\right)\right]$, Gradshteyn and Ryzhik [14], then,

$$
\begin{aligned}
f\left(x_{u v}\right) & =\sum_{p=0}^{v-u} \frac{(-1)^{p}\left(\begin{array}{c}
v-u \\
p
\end{array}\right)\left(\frac{1}{1-\alpha}\right)^{u+p-1}}{B(u, v-u+1)}\left[\sum_{q=0}^{\infty} w_{q}^{*} G^{q}\left(x_{u} ; \Lambda\right) g\left(x_{u} ; \Lambda\right)\right] \\
& \times \sum_{s=0}^{u+p-1}(-1)^{s}\left(\begin{array}{c}
u+p-1 \\
s
\end{array}\right)\left(\sum_{q=0}^{\infty} a_{q} G^{q}\left(x_{u} ; \Lambda\right)\right),
\end{aligned}
$$

where

$$
a_{0}=w_{0}^{s}, a_{m}=\frac{1}{m w_{0}} \sum_{q=1}^{m}(q s-m+q) w_{q} a_{m-q} ; m \geq 1,
$$

since, $\left(\sum_{q=0}^{\infty} w_{q}^{*} G^{q}\left(x_{u} ; \Lambda\right)\right)\left(\sum_{q=0}^{\infty} a_{q} G^{q}\left(x_{u} ; \Lambda\right)\right)=\left[\sum_{q=0}^{\infty} b_{q} G^{q}\left(x_{u} ; \Lambda\right)\right]$, Gradshteyn and Ryzhik [14], then,

$$
f\left(x_{u, v}\right)=\sum_{p=0}^{v-u} \frac{(-1)^{p}\left(\begin{array}{c}
v-u \\
p
\end{array}\right)\left(\frac{1}{1-\alpha}\right)^{u+p-1}}{B(u, v-u+1)} \sum_{s=0}^{u+p-1}(-1)^{s}\left(\begin{array}{c}
u+p-1 \\
s
\end{array}\right) g\left(x_{u} ; \Lambda\right)\left[\sum_{q=0}^{\infty} b_{q} G^{q}\left(x_{u} ; \Lambda\right)\right],
$$


where

$$
b_{m}=\sum_{q=0}^{m} w_{q}^{*} a_{m-q},
$$

hence,

$$
f\left(x_{u v}\right)=\sum_{q=0}^{\infty} d_{q} g\left(x_{u} ; \Lambda\right) G^{q}\left(x_{u} ; \Lambda\right)
$$

where

$$
d_{q}=\sum_{p=0}^{v-u} \frac{(-1)^{p}\left(\begin{array}{c}
v-u \\
p
\end{array}\right)\left(\frac{1}{1-\alpha}\right)^{u+p-1}}{B(u, v-u+1)} \sum_{s=0}^{u+p-1}(-1)^{s}\left(\begin{array}{c}
u+p-1 \\
s
\end{array}\right) b_{q} .
$$

The order statistics $r$-th moment of the $A K$ class of distributions can be written as

$$
E\left(\begin{array}{c}
x_{u: v}^{r} \\
)
\end{array}\right)=\int_{x_{u}} x_{u}^{r} f\left(x_{u: v}\right) d x_{u}
$$

substituting (14) into last equation yields

$$
E\left(X_{u v v}^{r}\right)=\sum_{q=o}^{\infty} d_{q} \int_{-\infty}^{\infty} X_{u}^{r} g\left(x_{u} ; \Lambda\right) G^{q}\left(x_{u} ; \Lambda\right) d x_{u},
$$

hence,

$$
E\left(X_{u, v}^{r}\right)=\sum_{q=o}^{\infty} d_{q} \tau_{r, q, o}
$$

\section{Estimation for Parameters of the $A K$ Class of Distributions Using MLE Method}

Let $X_{1}, X_{2}, \ldots, X_{n}$ be iid random variables following any $A K$ generalized distribution $(x ; \Delta)$ and the vector of parameter $\Delta=(\alpha, \beta, \theta, \Lambda)$ likelihood function [18] can be written as

$$
L(x)=\left(\frac{\beta \theta}{\alpha-1}\right)^{n}(\log \alpha)^{n} \prod_{i=1}^{n} g\left(x_{i} ; \Lambda\right) \prod_{i=1}^{n} G^{\beta-1}\left(x_{i} ; \Lambda\right) \prod_{i=1}^{n}\left(1-G^{\beta}\left(x_{i} ; \Lambda\right)\right)^{\theta-1} \prod_{i=1}^{n} \alpha^{1-\left(1-G \beta\left(x_{i} ; \Lambda\right)\right)^{\theta}},
$$

the log likelihood function is given by

$$
\begin{aligned}
\ell(x) & =n \log \frac{\beta \theta}{\alpha-1}+n \log (\log \alpha)+\sum_{i=1}^{n} \log g\left(x_{i} ; \Lambda\right)+(\beta-1) \sum_{i=1}^{n} \log G\left(x_{i} ; \Lambda\right) \\
& +(\theta-1) \sum_{i=1}^{n} \log \left(1-G^{\beta}\left(x_{i} ; \Lambda\right)\right)+(\log \alpha) \sum_{i=1}^{n}\left[1-\left(1-G^{\beta}\left(x_{i} ; \Lambda\right)\right)^{\theta}\right],
\end{aligned}
$$

the score functions for the parameters $\alpha, \beta, \theta$ and $\Lambda$ can be obtained by 


$$
\begin{gathered}
\frac{\partial \ell(x)}{\partial \alpha}=\frac{n}{1-\alpha}+\frac{n}{\alpha \log \alpha}+\frac{1}{\alpha} \sum_{i=1}^{n}\left[1-\left(1-G^{\beta}\left(x_{i} ; \Lambda\right)\right)^{\theta}\right], \\
\frac{\partial \ell(x)}{\partial \beta}=\frac{n}{\beta}+(\log \alpha) \theta \sum_{i=1}^{n} G^{\beta}\left(x_{i} ; \Lambda\right)\left[1-G^{\beta}\left(x_{i} ; \Lambda\right)\right]^{\theta-1}\left[\log G\left(x_{i} ; \Lambda\right)\right]+\sum_{i=1}^{n} \log G\left(x_{i} ; \Lambda\right) \\
-(\theta-1) \sum_{i=1}^{n} \frac{\left[G^{\beta}\left(x_{i} ; \Lambda\right) \log G\left(x_{i} ; \Lambda\right)\right]}{1-G^{\beta}\left(x_{i} ; \Lambda\right)}, \\
\frac{\partial \ell(x)}{\partial \theta}=\frac{n}{\theta}-(\log \alpha) \sum_{i=1}^{n}\left[1-G^{\beta}\left(x_{i} ; \Lambda\right)\right]^{\theta} \log \left[1-G^{\beta}\left(x_{i} ; \Lambda\right)\right]+\sum_{i=1}^{n} \log \left[1-G^{\beta}\left(x_{i} ; \Lambda\right)\right]
\end{gathered}
$$

and

$$
\begin{aligned}
\frac{\partial \ell(x)}{\partial \Lambda} & =(\log \alpha) \beta \theta \sum_{i=1}^{n}\left[1-G^{\beta}\left(x_{i} ; \Lambda\right)\right]^{\theta-1} G^{\beta-1}\left(x_{i} ; \Lambda\right) \frac{\partial G\left(x_{i} ; \Lambda\right)}{\partial \Lambda}+\sum_{i=0}^{n} \frac{1}{g\left(x_{i} ; \Lambda\right)} \frac{\partial g\left(x_{i} ; \Lambda\right)}{\partial \Lambda} \\
& +\sum_{i=0}^{n} \frac{(\beta-1)}{G\left(x_{i} ; \Lambda\right)} \frac{\partial G\left(x_{i} ; \Lambda\right)}{\partial \Lambda}-\sum_{i=0}^{n} \beta(\theta-1) \frac{G^{\beta-1}\left(x_{i} ; \Lambda\right)}{\left(1-G^{\beta}\left(x_{i} ; \Lambda\right)\right)} \frac{\partial G\left(x_{i} ; \Lambda\right)}{\partial \Lambda} .
\end{aligned}
$$

\section{A Simulation Study}

In this study, MLES for parameters of the $A K E$ distribution are obtained using random numbers to study the $M L E s$ finite sample behavior via the bootstrapping resample approach. Obtaining parameters estimates algorithm is detailed in the following steps:

Step (1): Generating a random sample $X_{1}, X_{2}, \ldots, X_{n}$ of sizes $n=(5,15,30,50,100,300)$ using the $A K E$ distribution.

Step (2): Selecting parameters different set values as: $\operatorname{set}(1):(\alpha=2, \beta=0.5, \theta=0.5, \lambda=0.2)$, set (2): ( $\alpha=2, \beta=0.5, \theta=0.5, \lambda=0.5)$ and set (3): ( $\alpha=2, \beta=0.5, \theta=0.5, \lambda=2$ ).

Step (3): Solving normal equations of the $A K E$ distribution via iteration to estimate distribution parameters.

Step (4): Replacing set (1), set(2) and set (3) with its estimators and repeating step (3) to compute, biases, $M L E S, R M S E$ (the root of mean squared error) and the Pearson type [19] of parameters estimators of the $A K E$ distribution.

Step (5): Repeating step (1) to step (4), 10000 times.

In this study, the conjugate gradient iteration method is performed in order to generate random numbers samples using Mathcad package v15. All results are included in tables and indicated in the appendix.

From study results, indicated in the appendix, one can see that: When sample size increases, biases, estimators, and RMSEs decrease. As sample size increases, the estimators can be consistent. $\hat{\alpha}$ and $\hat{\lambda}$ sampling distributions can be the Pearson type IV distribution in all times, $\hat{\beta}$ and $\hat{\theta}$ sampling 
distributions differ according to sample size. When $\lambda$ increases, for fixed values of $\alpha$ and $\beta$, the biases and MSES of $\hat{\alpha}$ and $\hat{\beta}$ decrease.

\section{Application}

A real data set is given using the MLE method to study the new model performance by Mathematica package version 10, some distributions are used as: The $A K E$ distribution, the gamma distribution (2 parameters), the normal distribution, the Weibull distribution, the Kumaraswamy exponential $(K E)$ distribution, the alpha power - power exponential (APE) distribution and the power exponential (PE) distribution. The following data set represents the classic lamps lifetime (Hours) for 40 devices, the data are given from the UK National Physical Laboratory, http://www.npl.co.uk/, $1.104,0.285,0.627,0.282,0.542,0.439,0.884,1.021,0.281,0.737,0.504,0.713,1.186,0.380,0.583,0.769$, $0.334,1.030,0.242,0.386,0.936,1.313,0.263,0.508,0.654,0.481,0.353,0.614,0.736,0.727,0.264,0.528$, $0.588,0.535,0.590,0.420,0.458,0.278,0.405,0.381$

Some goodness of fit measures results are indicated in the table (1), the likelihood ratio tests results are indicated in the table (2), the figure (3) describes probability density functions for some distributions having skewness and kurtosis values similar to the $A K E$ distribution (the gamma distribution with 2 parameters, the Normal distribution, the Weibull distribution), the figure (4) shows the empirical $C D F$ compared to $C D F s$ for some distributions (the gamma distribution with 2 parameters, the normal distribution, the Weibull distribution) and the figure (5) describes the probability density functions for special cases from the $A K E$ distribution (the Kumaraswamy exponential $(K E)$ distribution, the alpha power - power exponential (APE) distribution and the power exponential (PE) distribution).

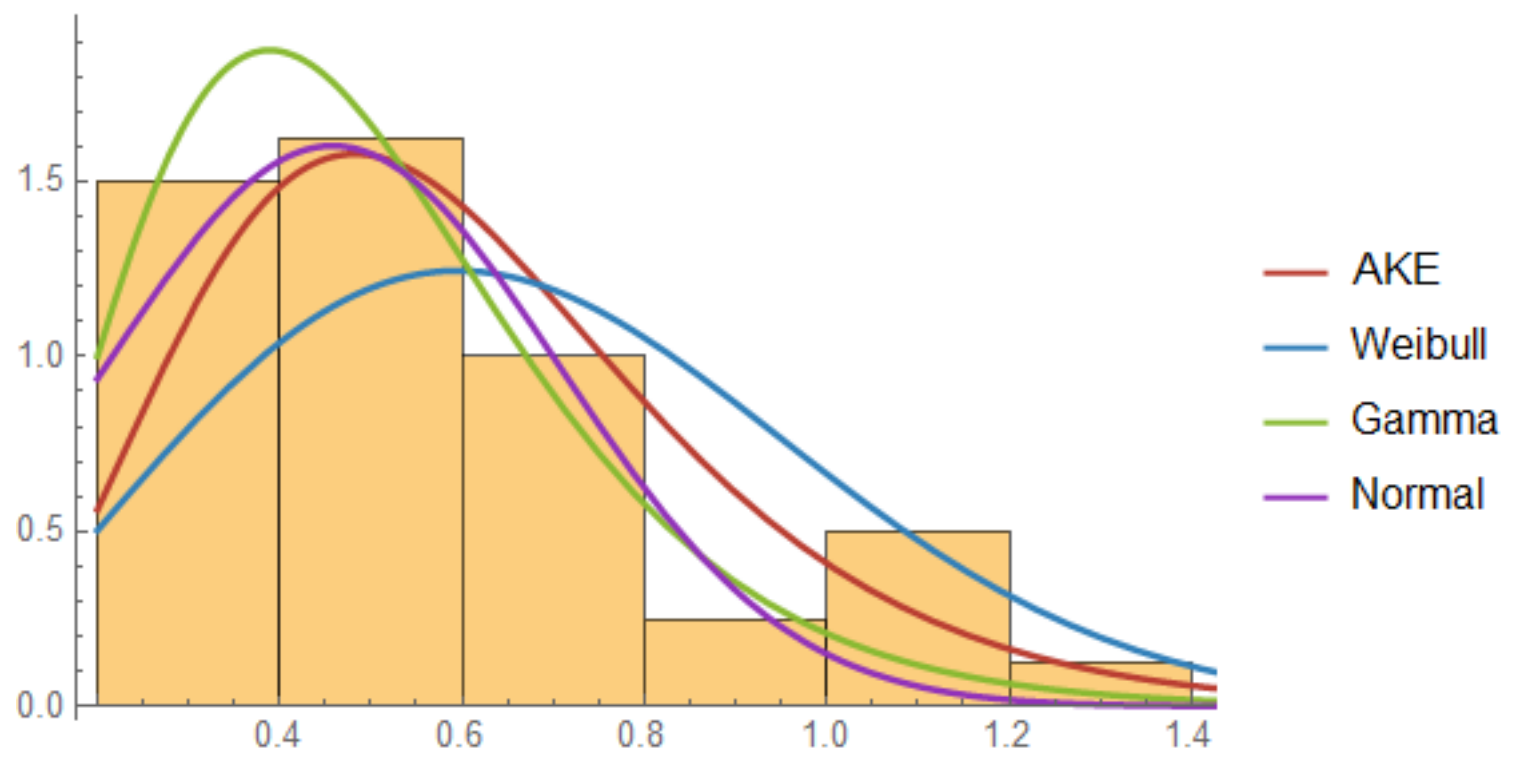

Figure 3: Probability density functions for some distributions having skewness and kurtosis 


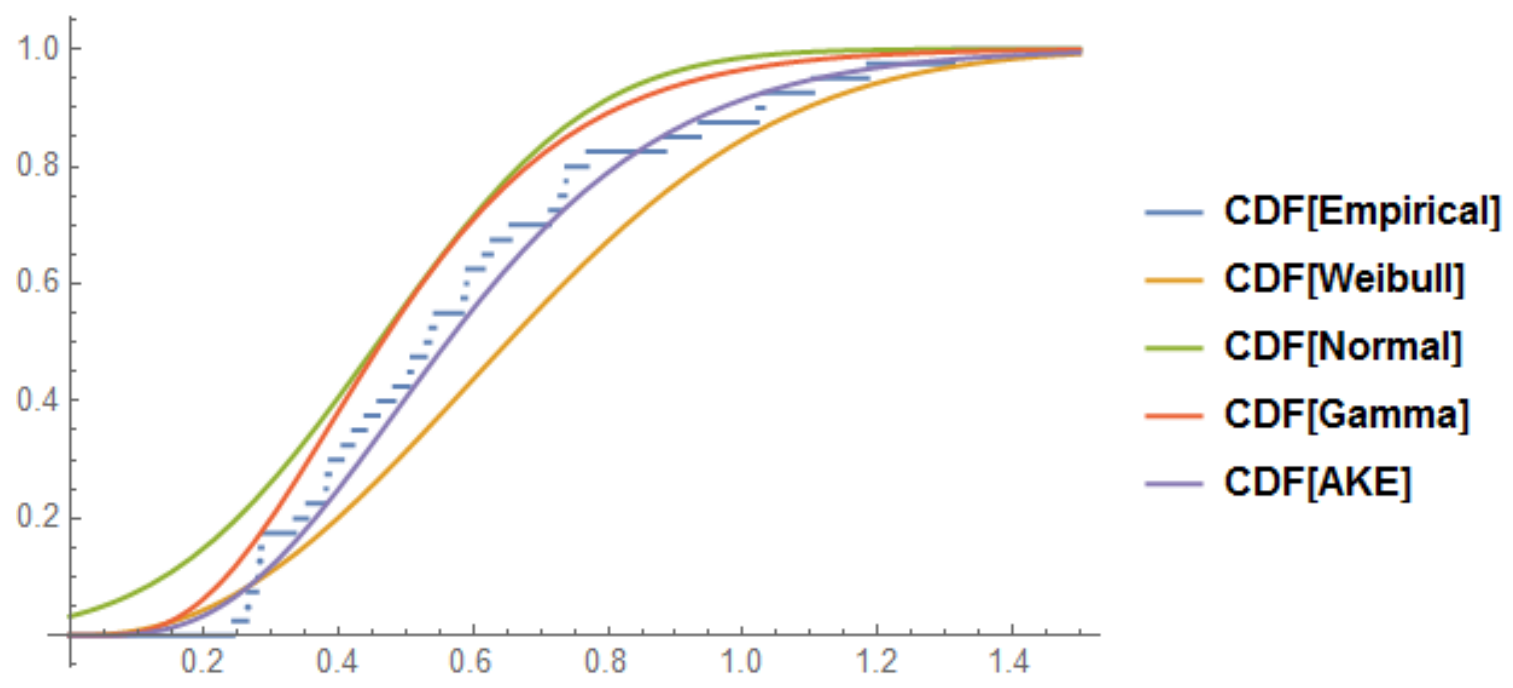

Figure 4: The empirical CDF compared to $C D F$ s for some distributions

Table 1: The parameter(s) MLE and the associated AIC and BIC values.

\begin{tabular}{|c|c|c|c|c|c|c|c|c|c|c|c|c|}
\hline \multirow{2}{*}{ Distribution } & \multicolumn{4}{|c|}{ MLE_parameters } & \multirow{2}{*}{$\begin{array}{l}\text { D) } \\
\sum_{0}^{\infty} \\
0 \\
\infty \\
\infty\end{array}$} & \multirow{2}{*}{ 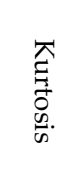 } & \multirow{2}{*}{ KS } & \multirow{2}{*}{ 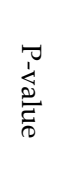 } & \multirow{2}{*}{$\begin{array}{c}\text { Log } \\
\text { Likel-ihood }\end{array}$} & \multirow{2}{*}{ AIC } & \multirow{2}{*}{ BIC } & \multirow{2}{*}{ CAIC } \\
\hline & $\alpha$ & $\beta$ & $\theta$ & $\lambda$ & & & & & & & & \\
\hline \multirow[t]{2}{*}{$A K-E$} & 2 & 4 & 3 & 2 & 0.058 & 2.403 & 0.08 & 0.938 & -0.555 & 7.111 & 8.488 & 7.777 \\
\hline & $(0.013)$ & $(0.033)$ & $(0.247)$ & $(0.182)$ & & & & & & & & \\
\hline \multirow[t]{2}{*}{ Weibull } & 2.307 & 0.762 & - & - & 0.451 & 2.961 & 0.203 & 0.062 & -3.901 & 11.802 & 15.180 & 12.127 \\
\hline & $(0.272)$ & $(0.048)$ & & & & & & & & & & \\
\hline \multirow[t]{2}{*}{ Gamma } & 4.503 & 0.111 & - & - & 0.942 & 4.332 & 0.144 & 0.338 & -2.649 & 9.299 & 12.677 & 9.624 \\
\hline & (1.088) & $(0.026)$ & & & & & & & & & & \\
\hline \multirow[t]{2}{*}{ Normal } & 0.458 & 0.249 & - & - & 0 & 3 & 0.193 & 0.086 & -10.05 & 24.115 & 27.492 & 24.439 \\
\hline & $(0.042)$ & $(0.0303)$ & & & & & & & & & & \\
\hline
\end{tabular}

In table 1, the distributions parameters $M L E$ s, parameters standard error (SEs), in parentheses, Kolmogorov-Smirnov (KS) test statistic, AIC (Akaike Information Criterion), CAIC (the consistent Akaike Information Criterion) and BIC (Bayesian information criterion), Merovcia and Puka [20], are computed for distributions having similar skewness and kurtosis values. Since, the $A K E$ distribution has the smallest KS, AIC, CAIC, BIC, SEs and the largest p-value, hence the AKE distribution can be the best fitted distribution to the data compared with other distributions which have similar skewness and kurtosis values. 


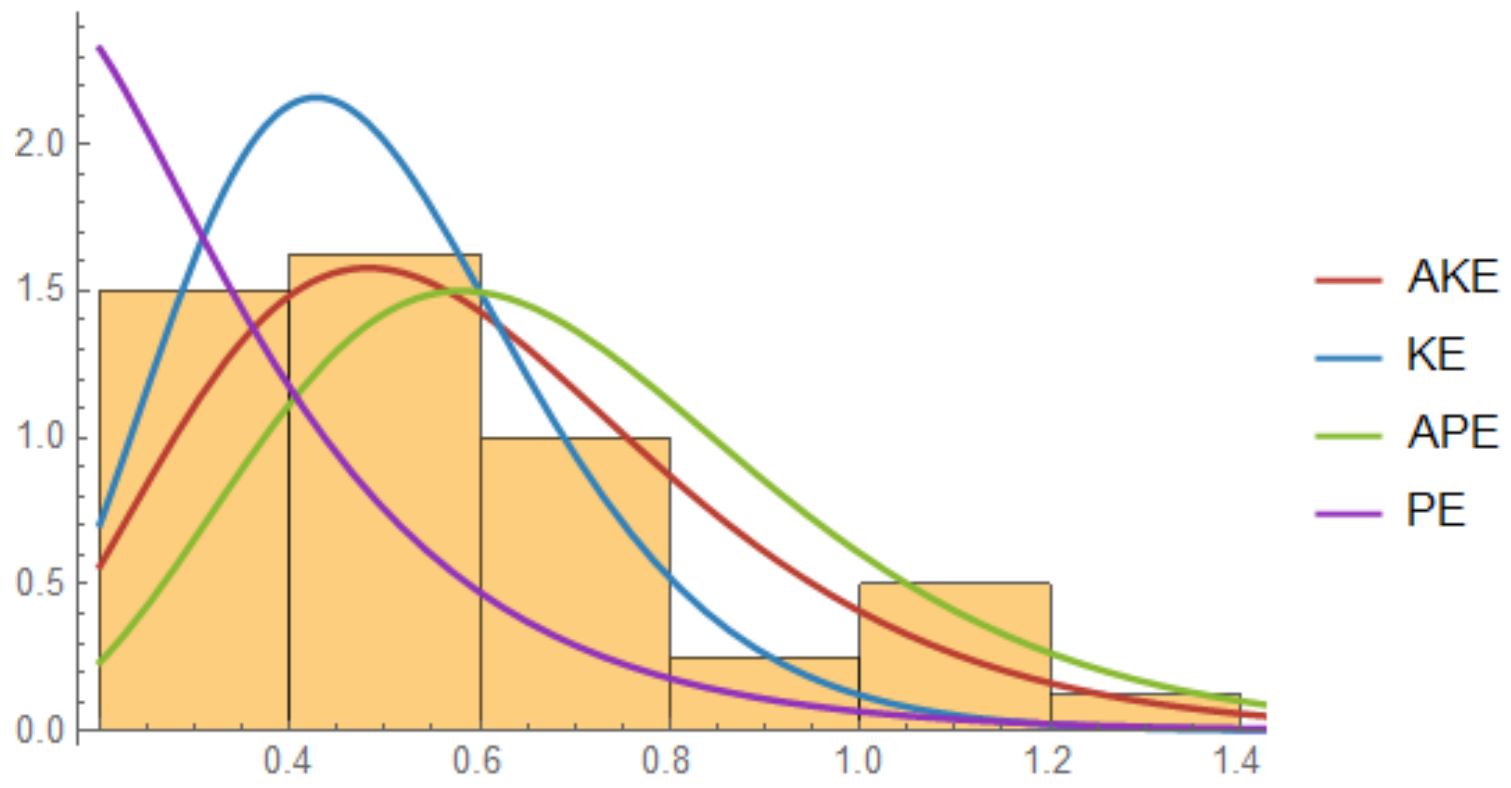

Figure 5: Probability density functions for special cases from the $A K E$ distribution

Table 2: The log-likelihood function, the likelihood ratio tests statistic and p-values.

\begin{tabular}{|c|c|c|c|c|c|c|c|c|}
\hline \multirow[b]{2}{*}{ Distribution } & \multicolumn{4}{|c|}{ Parameters } & \multicolumn{2}{|l|}{$\ell$} & \multirow{2}{*}{$\begin{array}{c}\text { df } \\
\text { (degrees } \\
\text { of } \\
\text { freedom) }\end{array}$} & \multirow[b]{2}{*}{$\mathrm{p}$-value } \\
\hline & $\alpha$ & $\beta$ & $\theta$ & $\lambda$ & likelihood) & $\begin{array}{c}\text { likelihood } \\
\text { ratio } \\
\text { test } \\
\text { statistic) }\end{array}$ & & \\
\hline$K E$ & - & $\begin{array}{c}2.176 \\
(0.418)\end{array}$ & $\begin{array}{c}1.647 \\
(0.329)\end{array}$ & $\begin{array}{c}1.928 \\
(0.284)\end{array}$ & -8.214 & 15.318 & 1 & $9.085 \times 10^{-5}$ \\
\hline$A P E$ & $\begin{array}{c}1.275 \\
(0.214)\end{array}$ & $\begin{array}{c}3.217 \\
(1.517)\end{array}$ & - & $\begin{array}{c}1.099 \\
(0.314)\end{array}$ & -10.247 & 19.384 & 1 & $1.069 \times 10^{-5}$ \\
\hline$P E$ & - & $\begin{array}{c}7.782 \\
(2.567)\end{array}$ & - & $\begin{array}{c}4.626 \\
(0.684)\end{array}$ & -14.869 & 28.628 & 2 & $6.074 \times 10^{-7}$ \\
\hline
\end{tabular}

*Note that the AKE distribution log likelihood $=-0.555$

In table 2, upon the likelihood ratio test, the null hypothesis is the data follow the nested model and the alternative is the data follow the full model, where the KE distribution, the APE distribution and the $P E$ distribution are nested by $A K E$ distribution, one can see that, all null hypotheses can be rejected at significance level is 0.05 


\section{Conclusion}

The alpha power Kumaraswamy class has an explicit form gives more flexibility in mathematical properties and random number generating. The alpha power Kumaraswamy class generalizes some important classes of distributions as the Kumaraswamy class, the alpha power class and the power function class. The alpha power Kumaraswamy exponential distribution has wide applications in real data sets and in some cases it can be the best fitted distribution. Author encourages researchers to study more cases from that flexible class.

Competing interests: The author declare that he has no competing interests

Data and Material Availability: One can find the date set at: http://www.npl.co.uk/

Code availability: Not applicable

Acknowledgements: The author thanks anyone gave an advice or any comment for this manuscript.

\section{References}

[1] Mahdavi, A. \& Kundu, D. (2017). A new method for generating distributions with an application to exponential distribution. Communications in Statistics-Theory and Method, 46, 6543-6557 .

[2] Ahmed, M.A. (2020). On the alpha power Kumaraswamy distribution: Properties, simulation and application. Revista Colombiana de Estadística, 43, 285-313.

[3] Wahed, A.S. (2006). A general method of constructing extended families of distributions from an existing continuous class. Journal of Probability and Statistical Science, 4, 165-177.

[4] Kumaraswamy, P. (1980). A generalized probability density function for double- bounded random-processes. Journal of Hydrology, 46, 79-88.

[5] Cordeiro, G.M., \& de Castro, M. (2011). A new family of generalized distributions. Journal of Statistical Computation \& Simulation, 81, 883-898.

[6] Pescim, R.R., Cordeiro, G.M., Demétrio, C.G., Ortega, E.M. \& Nadarajah, S. (2012). The new class of Kummer beta generalized distributions. SORT-Statistics and Operations Research Transactions, 153-180.

[7] McDonald, J.B. (1984). Some generalized functions for the size distribution of income. Econometrica, 52, 647-664.

[8] Alexander, C, Cordeiro, G.M., Ortega, E.M.M. \& Sarabia J.M. (2012). Generalized beta-generated distributions. Comput Stat Data Anal., 56, 1880-1897.

[9] El-Sherpieny, E.A. \& Ahmed, M.A. (2014). On the kumaraswamy Kumaraswamy distribution. International Journal of Basic and Applied Sciences, 3, 372-381.

[10] Mahmoud, M.R., El-Sherpieny, E.A. \& Ahmed, M.A. (2015). The new Kumaraswamy Kumaraswamy family of generalized distributions with application. Pakistan Journal of Statistics and Operations Research, 11, 159-180.

[11] Meniconi, M. \& Barry, D. (1996). The power function distribution: A useful and simple distribution to assess electrical component reliability. Microelectronics Reliability, 36, 1207-1212.

[12] Johnson, N.L., Kotz, S. \& Balakrishnan, N. (1995). Continuous Univariate Distributions. John wiley and Sons, New York .

[13] Greenwood, J.A., Landwehr, J.M., Matalas, N.C. \& Wallis, J.R. (1979). Probability weighted moments definition and relation to parameters of several distributions expressable in inverse form. Water Resources Research, 15, 1049-1054.

[14] Gradshteyn, I.S. \& Ryzhik, I.M. (2000). Tables of Integrals, Series and Products.Academic Press, San Diego, CA. 
[15] Ali Ahmed, M. (2021). The new form Libby-Novick distribution. Communications in Statistics-Theory and Methods, 50, 540-559.

[16] Meeker, W.Q. \& Escobar, L.A. (1998). Statistical Methods for Reliability Data. John Wiley, New York.

[17] Arnold, B.C., Balakrishnan, N. \& Nagaraja, H.N. (1992). A First Course in Order Statistics. John Wiley and Sons, Inc., New York.

[18] Garthwait, P.H., Jolliffe, I.P. \& jones, B. (2002). Statistical Inference. Prentice HallInternational (UK) Limited, London.

[19] Pearson, K. (1895). Contributions to the mathematical theory of evolution. II. Skew variations in homogeneous material. Philosophical Transactions of the Royal Society of London, Series A, 186, 343-414.

[20] Merovcia, F. \& Puka, L. (2014). Transmuted Pareto distribution. Prob Stat Forum, 7, 1-111.

\section{Appendix}

Results of the simulation study for different data sets:

\begin{tabular}{|c|c|c|c|c|c|c|c|c|}
\hline \multicolumn{9}{|c|}{$\operatorname{Set}(1):(\alpha=2, \beta=0.5, \theta=0.5, \lambda=0.2)$} \\
\hline $\begin{array}{c}\text { Sample } \\
\text { Size }\end{array}$ & Parameters & $\begin{array}{c}\text { Mean of } \\
\text { Estimators }\end{array}$ & Biases & $\begin{array}{c}\text { Total } \\
\text { Bias }\end{array}$ & RMSE & $\begin{array}{c}\text { Total } \\
\text { RMSE }\end{array}$ & $\begin{array}{l}\text { Pearson } \\
\text { System } \\
\text { Coefficients }\end{array}$ & $\begin{array}{c}\text { Pearson } \\
\text { Type }\end{array}$ \\
\hline \multirow[t]{4}{*}{10} & $\alpha=2$ & 2.262 & 0.262 & 1.474 & 1.258 & 2.185 & 0.202 & IV \\
\hline & $\beta=0.5$ & 0.759 & 0.259 & & 0.75 & & 0.27 & IV \\
\hline & $\theta=0.5$ & 0.92 & 0.42 & & 0.436 & & -0.27 & $\mathbf{I}$ \\
\hline & $\lambda=0.2$ & 1.565 & 1.365 & & 1.563 & & 0.576 & IV \\
\hline \multirow[t]{4}{*}{20} & $\alpha=2$ & 2.144 & 0.144 & 1.054 & 1.235 & 1.683 & 0.392 & IV \\
\hline & $\beta=0.5$ & 0.602 & 0.102 & & 0.246 & & 0.827 & IV \\
\hline & $\theta=0.5$ & 0.844 & 0.344 & & 0.36 & & 0.054 & IV \\
\hline & $\lambda=0.2$ & 1.181 & 0.981 & & 1.058 & & 0.594 & IV \\
\hline \multirow[t]{4}{*}{30} & $\alpha=2$ & 2.14 & 0.14 & 0.898 & 1.103 & 1.462 & 0.461 & IV \\
\hline & $\beta=0.5$ & 0.568 & 0.068 & & 0.173 & & -0.312 & I \\
\hline & $\theta=0.5$ & 0.808 & 0.308 & & 0.322 & & -0.496 & I \\
\hline & $\lambda=0.2$ & 1.03 & 0.83 & & 0.888 & & 0.14 & IV \\
\hline \multirow[t]{4}{*}{50} & $\alpha=2$ & 2.106 & 0.106 & 0.760 & 0.905 & 1.211 & 0.438 & IV \\
\hline & $\beta=0.5$ & 0.537 & 0.037 & & 0.118 & & -2.505 & I \\
\hline & $\theta=0.5$ & 0.776 & 0.276 & & 0.289 & & 0.021 & IV \\
\hline & $\lambda=0.2$ & 0.9 & 0.7 & & 0.742 & & 0.096 & IV \\
\hline \multirow[t]{4}{*}{100} & $\alpha=2$ & 2.073 & 0.073 & 0.224 & 0.368 & 0.391 & 0.44 & IV \\
\hline & $\beta=0.5$ & 0.511 & 0.011 & & 0.085 & & -0.267 & I \\
\hline & $\theta=0.5$ & 0.637 & 0.137 & & 0.051 & & 0.011 & IV \\
\hline & $\lambda=0.2$ & 0.362 & 0.162 & & 0.091 & & 0.666 & IV \\
\hline \multirow[t]{4}{*}{300} & $\alpha=2$ & 2.043 & 0.043 & 0.050 & 0.055 & 0.087 & 0.105 & IV \\
\hline & $\beta=0.5$ & 0.507 & 0.007 & & 0.012 & & -0.043 & I \\
\hline & $\theta=0.5$ & 0.506 & 0.006 & & 0.018 & & -3.643 & I \\
\hline & $\lambda=0.2$ & 0.225 & 0.025 & & 0.064 & & 0.57 & IV \\
\hline
\end{tabular}




\begin{tabular}{|c|c|c|c|c|c|c|c|c|}
\hline \multicolumn{9}{|c|}{$\operatorname{Set}(2):(\alpha=2, \beta=0.5, \theta=0.5, \lambda=0.8)$} \\
\hline $\begin{array}{l}\text { Sample } \\
\text { Size }\end{array}$ & Parameters & $\begin{array}{l}\text { Mean of } \\
\text { Estimators }\end{array}$ & Biases & $\begin{array}{l}\text { Total } \\
\text { Bias }\end{array}$ & RMSE & $\begin{array}{l}\text { Total } \\
\text { RMSE }\end{array}$ & $\begin{array}{l}\text { Pearson } \\
\text { System } \\
\text { Coefficients }\end{array}$ & $\begin{array}{l}\text { Pearson } \\
\text { Type }\end{array}$ \\
\hline \multirow[t]{4}{*}{10} & $\alpha=2$ & 2.239 & 0.239 & 3.506 & 1.234 & 6.406 & 0.203 & IV \\
\hline & $\beta=0.5$ & 0.743 & 0.243 & & 0.460 & & 0.268 & IV \\
\hline & $\theta=0.5$ & 0.959 & 0.459 & & 0.435 & & -0.372 & $\mathbf{I}$ \\
\hline & $\lambda=0.8$ & 4.26 & 3.46 & & 6.255 & & 0.583 & IV \\
\hline \multirow[t]{4}{*}{20} & $\alpha=2$ & 2.125 & 0.125 & 2.954 & 1.053 & 4.382 & 0.395 & IV \\
\hline & $\beta=0.5$ & 0.583 & 0.083 & & 0.126 & & 0.46 & IV \\
\hline & $\theta=0.5$ & 0.894 & 0.394 & & 0.391 & & 0.062 & IV \\
\hline & $\lambda=0.8$ & 3.724 & 2.924 & & 4.234 & & 0.592 & IV \\
\hline \multirow[t]{4}{*}{30} & $\alpha=2$ & 2.117 & 0.117 & 2.352 & 1.002 & 3.709 & 0.461 & IV \\
\hline & $\beta=0.5$ & 0.549 & 0.049 & & 0.101 & & -0.281 & I \\
\hline & $\theta=0.5$ & 0.878 & 0.378 & & 0.351 & & -0.221 & I \\
\hline & $\lambda=0.8$ & 3.118 & 2.318 & & 3.553 & & 0.692 & IV \\
\hline \multirow[t]{4}{*}{50} & $\alpha=2$ & 2.104 & 0.104 & 1.827 & 0.704 & 1.382 & 0.438 & IV \\
\hline & $\beta=0.5$ & 0.526 & 0.026 & & 0.091 & & -4.266 & I \\
\hline & $\theta=0.5$ & 0.796 & 0.296 & & 0.20 & & 0.018 & IV \\
\hline & $\lambda=0.8$ & 2.6 & 1.8 & & 1.169 & & 0.095 & IV \\
\hline \multirow[t]{4}{*}{100} & $\alpha=2$ & 2.053 & 0.053 & 1.25 & 0.171 & 0.470 & 0.439 & IV \\
\hline & $\beta=0.5$ & 0.509 & 0.009 & & 0.064 & & -1.288 & I \\
\hline & $\theta=0.5$ & 0.733 & 0.233 & & 0.267 & & 0.018 & IV \\
\hline & $\lambda=0.8$ & 2.036 & 1.236 & & 0.341 & & 0.046 & IV \\
\hline \multirow[t]{4}{*}{300} & $\alpha=2$ & 2.031 & 0.031 & 0.061 & 0.027 & 0.111 & 0.0018 & IV \\
\hline & $\beta=0.5$ & 0.504 & 0.004 & & 0.009 & & -0.0021 & I \\
\hline & $\theta=0.5$ & 0.521 & 0.021 & & 0.037 & & 0.00115 & IV \\
\hline & $\lambda=0.8$ & 0.848 & 0.048 & & 0.101 & & 0.529 & IV \\
\hline
\end{tabular}

$\operatorname{Set}(3):(\alpha=2, \beta=0.5, \theta=0.5, \lambda=2)$

\begin{tabular}{lllllllll}
\hline $\begin{array}{l}\text { Sample } \\
\text { Size }\end{array}$ & Parameters & $\begin{array}{l}\text { Mean } \\
\text { Estimators }\end{array}$ & Biases & $\begin{array}{l}\text { Total } \\
\text { Bias }\end{array}$ & RMSE & $\begin{array}{l}\text { Total } \\
\text { RMSE }\end{array}$ & $\begin{array}{l}\text { Pearson } \\
\text { System } \\
\text { Coefficients }\end{array}$ & $\begin{array}{l}\text { Pearson } \\
\text { Type }\end{array}$ \\
\hline 10 & $\alpha=2$ & 2.218 & 0.218 & 13.667 & 1.202 & 15.709 & 0.2 & IV \\
& $\beta=0.5$ & 0.727 & 0.227 & & 0.384 & & 0.279 & IV \\
& $\theta=0.5$ & 0.972 & 0.472 & & 0.518 & & -0.231 & I \\
& $\lambda=2$ & 15.656 & 13.656 & & 15.65 & & 0.561 & IV \\
20 & $\alpha=2$ & 2.113 & 0.113 & 9.814 & 1.004 & 10.634 & 0.393 & IV \\
& $\beta=0.5$ & 0.561 & 0.061 & & 0.117 & & 0.308 & IV \\
& $\theta=0.5$ & 0.897 & 0.397 & & 0.438 & & 0.06 & IV \\
& $\lambda=2$ & 11.806 & 9.806 & & 10.577 & & 0.605 & IV \\
30 & $\alpha=2$ & 2.095 & 0.095 & 8.307 & 0.809 & 8.934 & 0.454 & IV
\end{tabular}




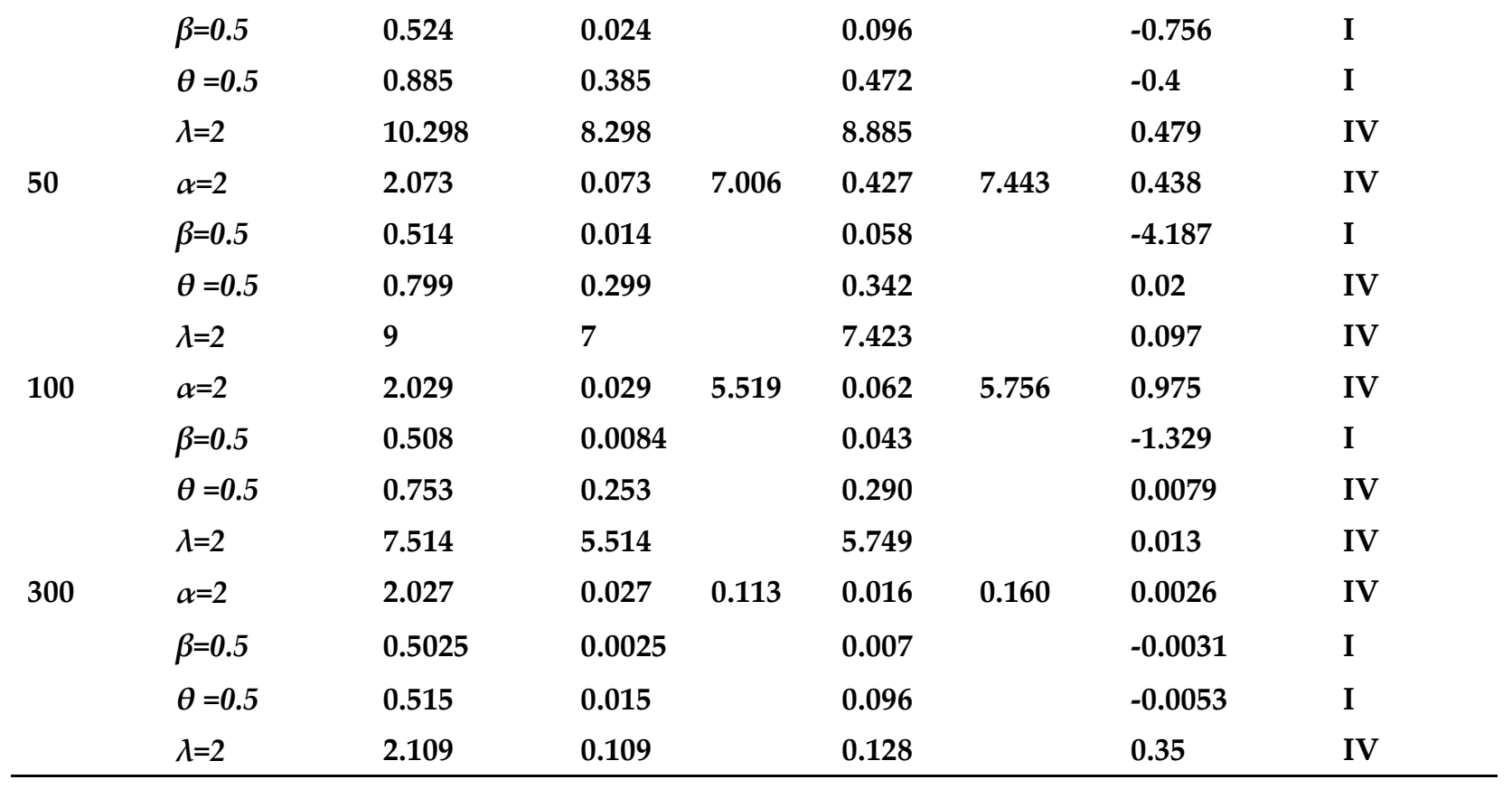

